\title{
Tuning surface plasmon polaritons in coaxial apertures
}

\author{
Linjuan Yang ${ }^{1}$, Tao Liu ${ }^{2}$, Jiangtao $\mathrm{Lv}^{1}$, Guangyuan $\mathrm{Si}^{1,}$, \\ ${ }^{1}$ College of Information Science and Engineering, Northeastern University, 110819, China \\ ${ }^{2}$ PetroChina Pipeline QHD Oil \& Gas Transportation Sub-Company, 066000, China \\ aemail: siguang0323@hotmail.com
}

Keywords: surface plasmon polaritons; tuning; nanophotonic devices

\begin{abstract}
Tuning of surface plasmons is important for developing nanophotonic devices. Coaxial apertures are emerging candidates for manipulating surface plasmon polaritons in the visible range. In this work, we show fine tuning of plasmons in coaxial nanoapertures. Since high transmittance can be achieved through coaxial apertures, it is useful to combine the optical response with other novel optical materials, leading to great potential for display techniques and filtering devices.
\end{abstract}

\section{Introduction}

Metamaterial (also known as the left handed material) was first proposed by Veselago[1] in 1968 and after that various useful applications triggered by metamaterials and surface plasmon polaritons (SPPs) such as superlensing[2], cloaking[3], perfect absorbing[4] have been thoroughly investigated and many devices with fascinating optical properties have been experimentally demonstrated. In addition, many other plasmonic nanocomponents have also been shown ranging from waveguiding to sensing thanks to the unique capability of SPPs. The main limitation of SPPs based on noble metals refers to the high energy loss. However[5], SPPs can be manipulated in different ways since the SPP modes of a nanostructure rest with its composition, scale and shape. Recently, coaxial aperture based nanorings have drawn increasing attention and interest due to their great potential in color filtering and generating negative refractive index in a wide frequency range[6]. Varying geometries have also been reported[7]. Further tuning of SPPs can be simply realized by finely controlling the structural parameters. Dynamic manipulation can also be achieved by employing materials with electro-optic effects such as lithium niobate and liquid crystals.

In this work, we investigate the gap plasmons in coaxial apertures in an optically thick metal film. Using finite-difference time-domain (FDTD) calculations, we found that it is feasible to manipulate the gap plasmons in coaxial apertures by precisely controlling the geometric parameters such as the gap width, periodicity and depth of the nano-apertures.

\section{Fabrication}

Figure 1 is the schematic illustration of the coaxial apertures under investigation in this work with all critical parameters labeled. Here, ra and rb are the inner and outer radius of the coaxial nanorings and $g$ and $p$ denote the gap width and the periodicity, respectively. One should note that during the discussion of the hybrid plasmon effects in these coaxial apertures, it is simplified to consider the gap plasmon effect only by fixing the periodicity at a constant value because the periodical plasmon effect can be suppressed to some extent. Typically, a 2D coaxial aperture array has two main resonances in the transmittance spectrum. One is caused by cylindrical surface plasmons and the other one is referred to planar surface plasmons. The former is mainly influenced by the structural parameters of the aperture, while the latter is highly dependent on the periodicity of the array. 


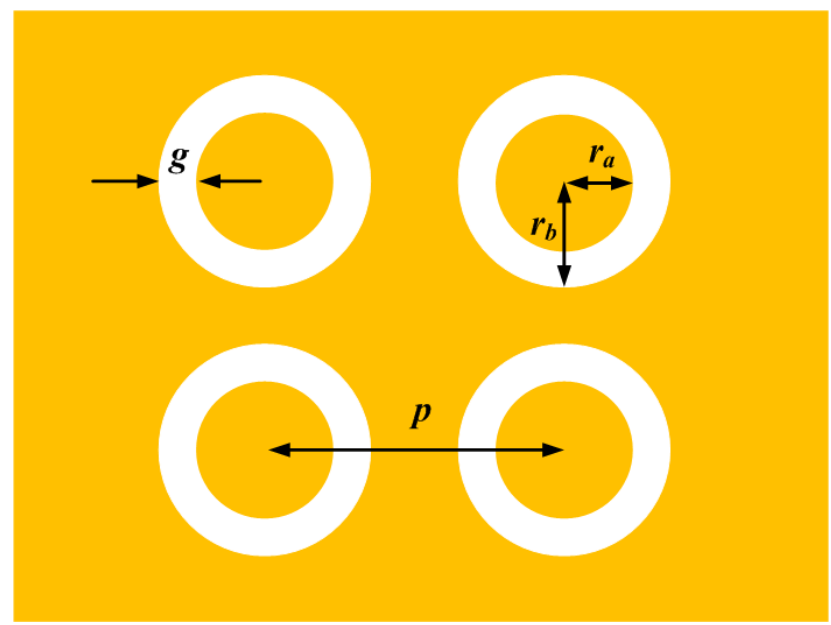

Fig.1. Schematic illustration of the coaxial apertures under investigation in this work.

\section{Results and discussion}

It is well-known that the transmittance through such coaxial apertures is due to the excitation of a guided mode inside them. This mode is the TE11-like mode that can be compared to the TE11 mode of a coaxial waveguide made in a perfect conductor. For $1 \mathrm{D}$ nanoslit configurations, transmittance resonance only happens for TM polarized light because no resonant effects can be observed under TE polarization. However, plasmonic coaxial nanostructures can support propagating modes due to the excitation of plasmons.

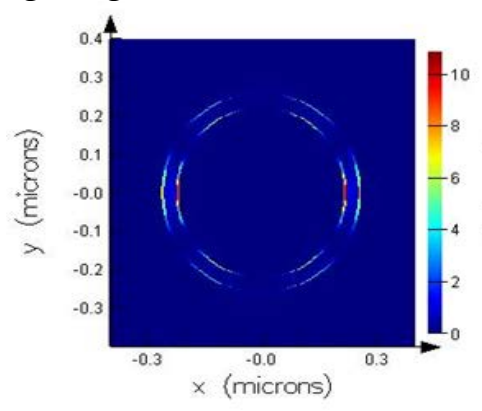

Ex

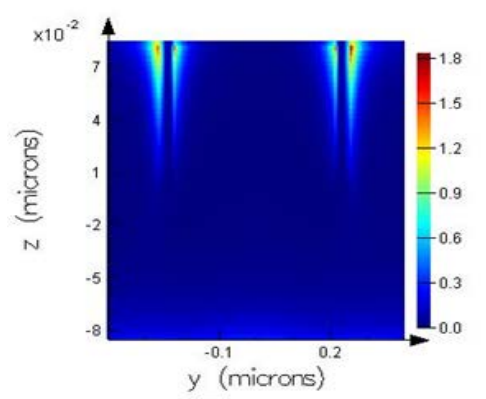

Ex

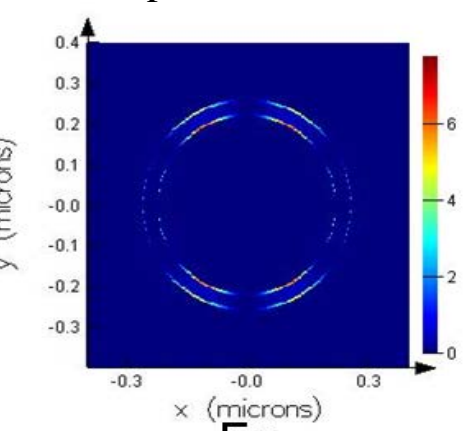

Ey

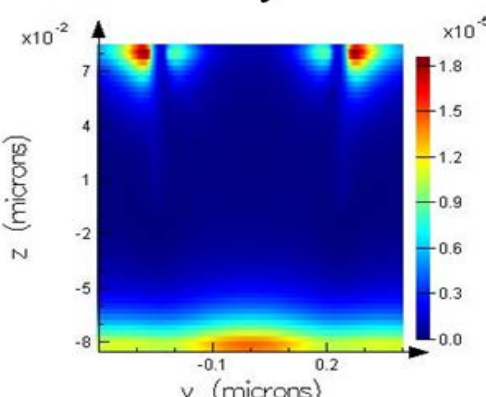

Hy

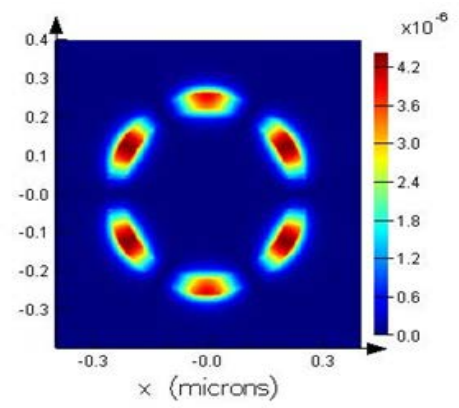

$\mathrm{Hz}$

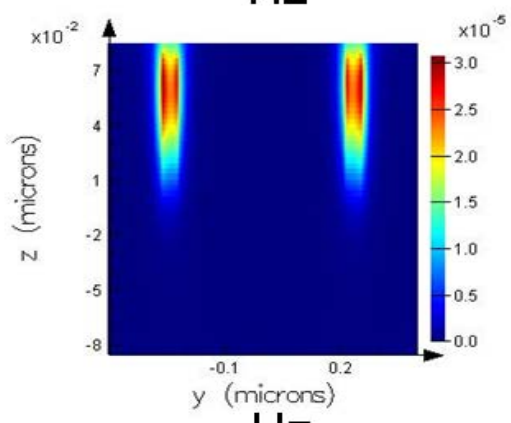

$\mathrm{Hz}$

Fig.2. Near field distribution for coaxial apertures with $80 \mathrm{~nm}$ depth. Top panel, top view. Bottom panel, cross-section view.

Our initial thoughts about using nanoantennae to construct optical filters were triggered by our previous work about wavelength-selective color devices. Such optical components take advantage of the novel properties of plasmonics which are barely available by using any other traditional methods or dielectric structures. One typical relevant work of other researchers is using helium ions to create bow-tie antennae with ultrasmall gaps. We have tried to import the real images of the nanoantennae and compare the new simulation results with the old ones which were obtained by drawing the structures in the simulator. However, the new calculated field distribution is not as good 
as expected. We believe this is because: 1 . the antennae surface is assumed to be smooth, which is different from the real case (fabricated sample has a rough topography due to redeposition during milling); 2. the profile (sidewalls, for instance) of the antennae is assumed to be vertical during simulations, which is also different from the real structures (oblique sidewalls).

Figure 3 demonstrates the calculated near field distributions of coaxial apertures with $160 \mathrm{~nm}$ depth. Selected transmission can be obtained through TE11 modes by engineering annular apertures with various geometrical designs. When the thickness of a metal film is fixed, different combinations of diameter values and gap sizes will generate different reflection phases through the annular aperture cavities, affecting the propagation mode inside the cavities and leading to selected transmission of certain modes. The gaps are not uniform in the simulations by importing the real structures because fabrication imperfections are almost inevitable in experiments. Therefore, the near-field distribution may be not as good as expected although the dimension parameters are the same with the real case.

The position of the plasmonic resonance can be well controlled by varying the dielectric function of the surrounding medium. The angle dependency can be measured by using a protractor placed between the plane of the substrate and the normal direction (vertical to the substrate). Although it was not a very accurate measurement compared with any other commercial measurement equipment, it was sufficient to evaluate the incident angle and observe the filtering effect with different orientations. Further reshaping process can be carried out using milling due to its versatile functionality. One can tilt the sample and treat the nanostructures from different angles, enabling efficient processing methods which can further lead to varying useful devices. More detailed results will be reported elsewhere after a thorough study.

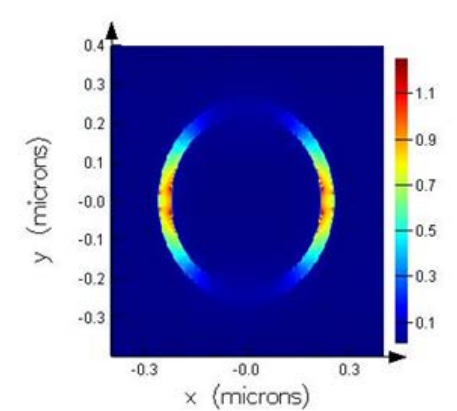

Ex

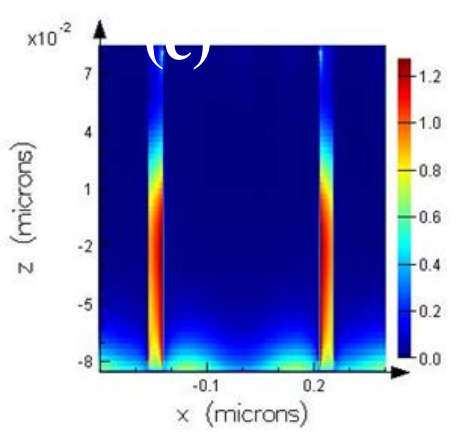

Ex

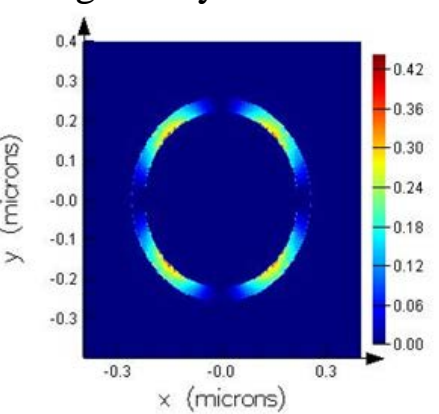

Ey

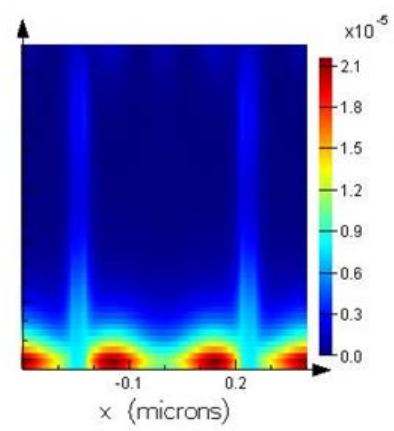

Hy

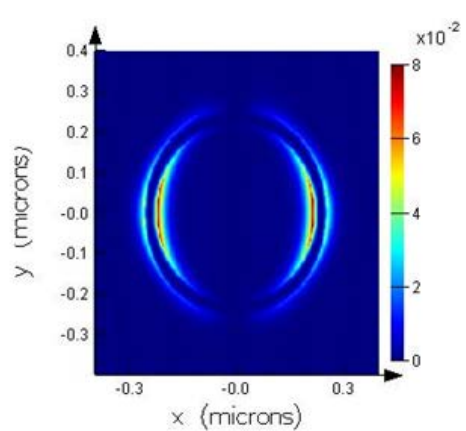

$\mathrm{Ez}$

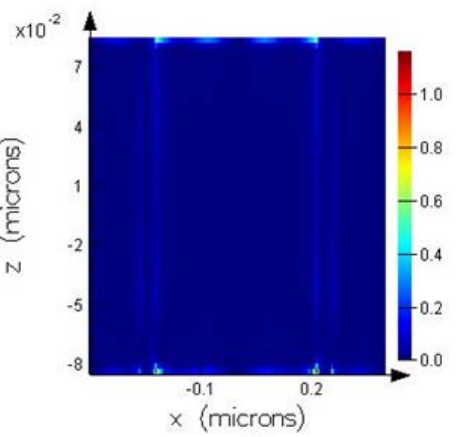

$\mathrm{Hz}$

Fig.3. Near field distribution for coaxial apertures with $160 \mathrm{~nm}$ depth. Top panel, top view. Bottom panel, cross-section view.

Theoretical results have confirmed that selected transmittance is the direct result of the TE11 mode inside the coaxial apertures. However, the second plasmonic mode is also observed in our design but it is not interesting for us because we are trying to avoid multi-transmission peaks which would disturb the purity of the output plasmon resonances obtained. One should also note that the good confinement of the intensity inside the cavities (between the inner and outer radii) is very important to guide different SPP modes and further adjust their properties, enabling new applications in photonics and optics. 


\section{Conclusion}

In summary, we have shown the near field distribution of coaxial apertures with different depths. Fine tuning of gap plasmons can be realized by controlling the geometric parameters of the coaxial apertures. Such nanoapertures are potentially useful for developing various devices in optics and nanophotonics since strong confinement of light in the gaps can be obtained.

\section{Acknowledgement}

This work was supported by the Natural Science Foundation of Hebei Province (Grant Nos. H2015501133 and F2014501127), Science and Technology Research Funds for Higher Education of Hebei Province (Grant No. ZD20132011), Specialized Research Fund for the Doctoral Program of Higher Education (Grant No. 20130042120048), Science and Technology Foundation of Liaoning Province (Grant No. 20131031), Science and Technology Research \& Development Project Funds of Shenzhen (Grant No. JCYJ20120618142137681), and the National Natural Science Foundation of China (Grant Nos. 61405031and 31170956), the science and technology planning project of Hebei province (13273303D).

\section{References}

[1] X. X. Jiang, Q. C. Gu, F. W. Wang, J. T. Lv, Z. H. Ma, G. Y. Si, Fabrication of coaxial plasmonic crystals by focused ion beam milling and electron-beam lithography, Mater. Lett., 100 (2013) 192-194.

[2] W. L. Barnes, A. Dereux, T. W. Ebbesen, Surface plasmon subwavelength optics, Nature 424 (2003) 824-830.

[3] Z. H. Huang, X. P. Wang, S. Y. Zhan, X. Liu, Contrast-enhancing polarization control method for surface plasmon imaging sensor, Opt. Eng., 51 (2012) 094402.

[4] G. Y. Si,, Y. H. Zhao, H. Liu, S. Teo, M. S. Zhang, T. Huang, A. J. Danner, J. H. Teng, Annular aperture array based color filter, Appl. Phys. Lett., 99 (2011) 033105.

[5] J. Francés, S. Bleda, M. Lázara Álvarez, F. Javier Martínez, A. Márquez, C. Neipp, A. Beléndez, Acceleration of split-field finite difference time-domain method for anisotropic media by means of graphics processing unit computing , Opt. Eng., 53 (2013) 011005.

[6] G. Y. Si,, Y. H. Zhao, J. T. Lv, M. Q. Lu, F. W. Wang, H. L. Liu, N. Xiang, T. Huang, T. J. Danner, J. H. Teng, Y. J. Liu, Reflective plasmonic color filters based on lithographically patterned silver nanorod arrays, Nanoscale, 5 (2013) 6243-6348.

[7] G. Y. Si, A. J. Danner, S. Teo, E. Teo, J. H. Teng, A. A. Bettiol, Photonic crystal structures with ultrahigh aspect ratio in lithium niobate fabricated by focused ion beam milling, J. Vac. Sci.Technol.B, 29 (2011) 021205-02109. 